\title{
Ground glass nodules with 5 years' stability can grow after 10- year follow-up: do genetic features determine the fate?
}

\author{
Yoshihisa Kobayashi $^{1,2}$, Tetsuya Mitsudomi ${ }^{1}$ \\ ${ }^{1}$ Department of Thoracic Surgery, Kindai University Faculty of Medicine, Osaka-Sayama, Japan; ${ }^{2}$ Department of Medical Oncology, Dana-Farber \\ Cancer Institute, Boston, USA \\ Correspondence to: Tetsuya Mitsudomi. Department of Thoracic Surgery, Kindai University Faculty of Medicine, 377-2 Ohno-Higashi, Osaka-Sayama \\ 589-8511, Japan. Email: mitsudom@med.kindai.ac.jp. \\ Provenance: This is an invited article commissioned by the Academic Editor Chenyang Dai, MD, PhD (Department of Thoracic Surgery, Shanghai \\ Pulmonary Hospital, School of Medicine, Tongji University, Shanghai, China). \\ Comment on: Lee HW, Jin KN, Lee JK, et al. Long-Term Follow-Up of Ground-Glass Nodules After 5 Years of Stability. J Thorac Oncol \\ 2019;14:1370-7.
}

Submitted Sep 30, 2019. Accepted for publication Oct 21, 2019.

doi: $10.21037 /$ tlcr.2019.10.16

View this article at: http://dx.doi.org/10.21037/tlcr.2019.10.16

Ground-glass nodules (GGNs) in the lung are lesions that appear hazy on computed tomography (CT), without obscuring underlying bronchial structures or pulmonary vessels. Both benign lesions including inflammation, hemorrhage, or focal interstitial fibrosis, and malignancies can present as GGNs. Slow-growing or stable GGNs indicate the presence of early stage lung cancers or preinvasive lesions, such as atypical adenomatous hyperplasia (AAH) and adenocarcinoma in situ (AIS) (1); these, along with lepidic predominant lung adenocarcinomas, grow along alveolar structures, maintain the air space and thus appear as GGNs on CT. GGNs are categorized into pure GGNs without solid components and part solid GGNs with solid components. AAH and AIS typically present as pure GGNs, whereas minimally invasive adenocarcinoma (MIA) and lepidic invasive adenocarcinoma are found as part solid GGNs, because their pathologically invasive parts appear solid on CT.

High-quality CT images with thin-section slices have been clinically available since the early 2000's. Between 2008 and 2013, five reports (including ours) have described the natural history of more than 100 GGNs, with median or mean follow-up periods of 2.4 to 4.9 years (2-6). Although the inclusion criteria and definition of growth in each of these reports vary, the overall data show that $16 \%$ of pure GGNs and $41 \%$ of part solid GGNs increase their size or solid components, whereas the rest did not change (7)
(Figure 1). Our paper underscores that the tendency of GGNs to grow is clear in all cases within the first 3 years; thus, we had suggested that a 3 -year follow-up is reasonable to distinguish these lesions, considering that the tumor doubling time of GGN is about 800 to 1,000 days (3).

In 2016, data of a prospective study conducted in 8 institutions were reported by Kakinuma et al. (8). As many as 1,229 GGNs in 795 patients were evaluated with a mean follow-up period of 4.3 years. The 2-mm growth probability in year 5 was $14 \%, 24 \%$, and $48 \%$ for pure, heterogeneous (part solid GGN in the lung window setting but the solid component disappears in mediastinal window setting) and part-solid GGNs, respectively. Some GGNs did begin to increase their size after 3-year follow-up. In 2017 the minimum period of following GGNs in the updated guidelines from the Fleischner Society was extended from 3 to 5 years (9). However, whether GGNs grow any further after the 5-year follow-up was left unclear. Unfortunately, further analyses of this prospective multi-institutional study, with longer follow-ups, were not preplanned in the protocol.

And now, a recent paper by Lee et al. reviews the natural history of GGNs with follow-up period of more than 10 years (10). They retrospectively evaluated GGNs that were pure or part solid. and $\leq 3 \mathrm{~cm}$ in diameter, without a limitation on the ratio of the solid part. All GGNs were detected incidentally during regular health checkups done 

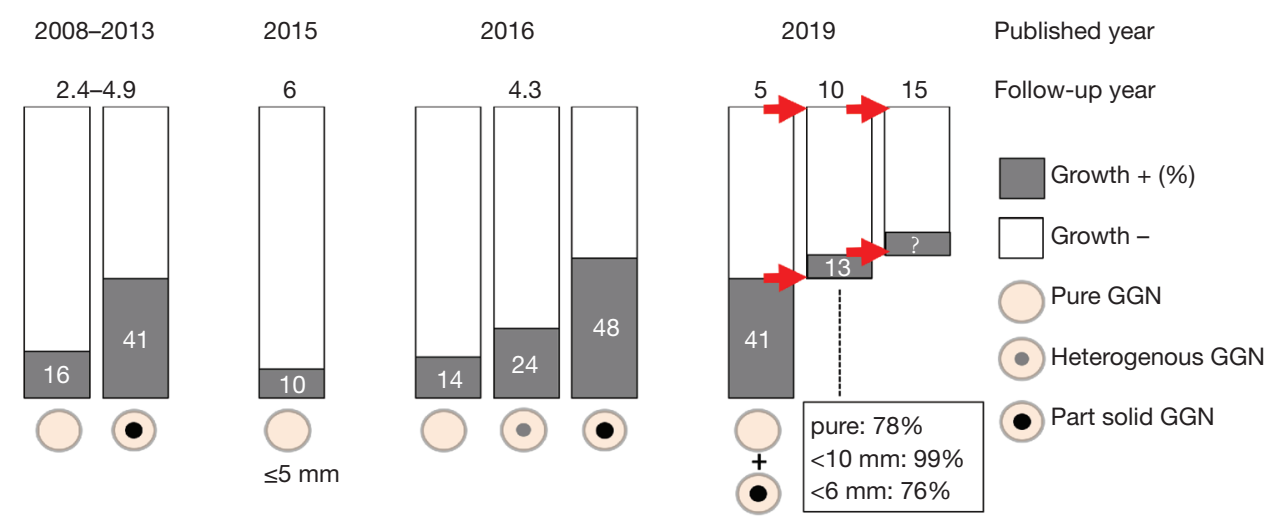

Figure 1 Natural history of ground glass nodules. The growth rates and median or mean follow-up period are summarized. Heterogenous GGN is defined as GGN with solid components only in the lung window but not in the mediastinal window setting (8). GGN, ground-glass nodule.

between 2003 and 2017. Of 351 GGNs from 242 patients, $41 \%$ [143] changed in size within the first 5 years and the remaining 59\% [208] were stable. The majority of the stable GGNs were small and pure: $99 \%$ were $<10 \mathrm{~mm}$ in diameter, $76 \%$ were $<6 \mathrm{~mm}$, and $78 \%$ were pure GGNs. During a 136 -month follow-up, an additional $13 \%$ of the GGNs (27/208) showed evidence of growth after 5 years of being stable (Figure 1): this is a remarkable number, even for a retrospective study from a single institution, and is similar to growth rates of conventionally reported pure GGNs (10-16\%) (Figure 1). The 5 year-stability factor seems to be highly selective for a small pure GGNs.

Collectively, these findings indicate that in clinical practice, we should not stop following GGNs, given the risk of overlooking potential invasive adenocarcinoma. Lee et al.'s report suggests that we should follow GGNs for at least 10 years. To avoid unnecessary CT examinations, an appropriate follow-up interval between imaging sessions should be established. Also, from Kakinuma's prospective study, the data on volume doubling times (VDTs) of resected GGNs show that median VDTs for AIS, MIA, and invasive adenocarcinoma are not statistically different, and are 811, 802, 631 days, respectively (8), while the VDTs for the solid component are 100, 223, 106 days, respectively. These indicate that part-solid GGNs should be more closely followed than pure GGNs. Also, that reported growth predictors such as size $(>10 \mathrm{~mm})$ and smoking history (or male gender) should be additionally considered $(8,11)$.

What happens to GGNs at 15 years? Lee and coworkers show that the characteristics of GGNs that had a 10 -year stability were as follows: $99 \%$ of them were $<10 \mathrm{~mm}$ in diameter, $77 \%$ of them were $<6 \mathrm{~mm}$, and $79 \%$ of them were pure GGNs (10). Based on these characteristics, we predict that approximately $10 \%$ of GGNs will grow between 10 to 15 years of follow-up-in other words, $90 \%$ of them will still be unchanged; and the cumulative growth rate does not seem to reach $100 \%$ even over a few decades.

We have evaluated and discussed the influence of genetic features on the growth of GGNs (12), and hypothesized that EGFR mutant GGNs will grow, whereas some of KRAS or BRAF mutant GGNs will not grow. Not all KRAS mutant AAHs may be homogenous and differences among these tumors may be derived from different cells of origin (13). We also suggest that unchanged AAH involves cancer immunoediting, a process whereby the immune system can constrain as well as promote tumor development. Cancer Immunoediting proceeds through 3 phases-elimination, equilibrium and escape (14)wherein tumor immunogenicity is edited, and immunosuppressive mechanisms that enable disease progression are acquired. We hypothesize that unchanged GGNs are in the equilibrium phase.

In conclusion, Lee et al. document that even if GGNs are stable for 5 years, $13 \%$ of them will grow during at least an additional 5 -year follow-up. Thus, we cannot stop following GGNs by CT but must establish an appropriate interval for CT examinations, based on the possibility of growth. Further genetic analyses could clarify the fate of GGNs. Over the next several years, we expect to obtain data on patients with a follow-up period of 15 years and establish the possibility of growth of the GGNs even after 
10 years of stability.

\section{Acknowledgments}

The authors are grateful to Dr. Sonal Jhaveri, Science Program Director of Postdoc and Graduate Student Affairs Office in Dana-Farber Cancer Institute, for editing a draft of this commentary.

\section{Footnote}

Conflicts of Interest: The authors have no conflicts of interest to declare.

Ethical Statement: The authors are accountable for all aspects of the work in ensuring that questions related to the accuracy or integrity of any part of the work are appropriately investigated and resolved.

\section{References}

1. Travis WD, Brambilla E, Noguchi M, et al. International association for the study of lung cancer/american thoracic society/european respiratory society international multidisciplinary classification of lung adenocarcinoma. J Thorac Oncol 2011;6:244-85.

2. Hiramatsu $M$, Inagaki $T$, Matsui $Y$, et al. Pulmonary ground-glass opacity (GGO) lesions-large size and a history of lung cancer are risk factors for growth. J Thorac Oncol 2008;3:1245-50.

3. Kobayashi Y, Fukui T, Ito S, et al. How long should small lung lesions of ground-glass opacity be followed? J Thorac Oncol 2013;8:309-14.

4. Matsuguma H, Mori K, Nakahara R, et al. Characteristics of subsolid pulmonary nodules showing growth during follow-up with CT scanning. Chest 2013;143:436-43.

Cite this article as: Kobayashi Y, Mitsudomi T. Ground glass nodules with 5 years' stability can grow after 10-year follow-up: do genetic features determine the fate? Transl Lung Cancer Res 2019;8(Suppl 4):S425-S427. doi:10.21037/tlcr.2019.10.16
5. Chang B, Hwang JH, Choi YH, et al. Natural history of pure ground-glass opacity lung nodules detected by lowdose CT scan. Chest 2013;143:172-8.

6. Lee SW, Leem CS, Kim TJ, et al. The long-term course of ground-glass opacities detected on thin-section computed tomography. Respir Med 2013;107:904-10.

7. Kobayashi Y, Mitsudomi T. Management of ground-glass opacities: should all pulmonary lesions with ground-glass opacity be surgically resected? Transl Lung Cancer Res 2013;2:354-63.

8. Kakinuma R, Noguchi M, Ashizawa K, et al. Natural History of Pulmonary Subsolid Nodules: A Prospective Multicenter Study. J Thorac Oncol 2016;11:1012-28.

9. MacMahon H, Naidich DP, Goo JM, et al. Guidelines for Management of Incidental Pulmonary Nodules Detected on CT Images: From the Fleischner Society 2017. Radiology 2017;284:228-43.

10. Lee HW, Jin KN, Lee JK, et al. Long-Term Follow-Up of Ground-Glass Nodules After 5 Years of Stability. J Thorac Oncol 2019;14:1370-7.

11. Kobayashi Y, Sakao Y, Deshpande GA, et al. The association between baseline clinical-radiological characteristics and growth of pulmonary nodules with ground-glass opacity. Lung Cancer 2014;83:61-6.

12. Kobayashi Y, Mitsudomi T, Sakao Y, et al. Genetic features of pulmonary adenocarcinoma presenting with groundglass nodules: the differences between nodules with and without growth. Ann Oncol 2015;26:156-61.

13. Kobayashi Y, Ambrogio C, Mitsudomi T. Groundglass nodules of the lung in never-smokers and smokers: clinical and genetic insights. Transl Lung Cancer Res 2018;7:487-97.

14. O'Donnell JS, Teng MWL, Smyth MJ. Cancer immunoediting and resistance to $\mathrm{T}$ cell-based immunotherapy. Nat Rev Clin Oncol 2019;16:151-67. 